\title{
Assessment of the cardiac safety between cetuximab and panitumumab as single therapy in Chinese chemotherapy-refractory mCRC
}

\author{
Xue-miao Tang, ${ }^{1,2, *}$ Hao \\ Chen, ${ }^{2,3, *}$ Qing Li, ${ }^{1,2}$ Yiling \\ Song, ${ }^{2,3}$ Shuping Zhang, ${ }^{2,3}$ \\ Xiao-Shuan $\mathrm{Xu},{ }^{4}$ Yiwei \\ $\mathrm{Xu},{ }^{5,6}$ Shulin $\mathrm{Chen}^{2,3}$ \\ 'Department of Ultrasound and \\ Electrocardiogram, ${ }^{2}$ State Key \\ Laboratory of Oncology in South \\ China, Collaborative Innovation \\ Center for Cancer Medicine, \\ ${ }^{3}$ Department of Clinical Laboratory, \\ Sun Yat-Sen University Cancer Center. \\ Guangzhou, ${ }^{4} \mathrm{School}$ of Laboratory \\ Medicine, Guangdong Medical \\ University, ${ }^{5}$ Department of Clinical \\ Laboratory, The Cancer Hospital \\ of Shantou University Medical \\ College, ${ }^{6}$ The Key Laboratory of \\ Molecular Biology for High Cancer \\ Incidence Coastal Chaoshan Area, \\ Shantou University Medical College, \\ Guangdong, China \\ *These authors contributed equally \\ to this work
}

Correspondence: Shulin Chen

Department of Clinical Laboratory, Sun Yat-Sen University Cancer Center, 65I Dongfeng Road East, Guangzhou 510060, China

Fax +86 2087343438

Email chenshl@sysucc.org.cn

Yiwei Xu

Department of Clinical Laboratory, The Cancer Hospital of Shantou University Medical College, The Key Laboratory of Molecular Biology for High Cancer Incidence Coastal Chaoshan Area, Shantou University Medical College, Number 22, Xinling Road, Shantou, Guangdong, 5I504I, China Fax +86 75488560352 Email yiwei5 I2@I26.com
This article was published in the following Dove Press journal: OncoTargets and Therapy

\begin{abstract}
Objective: The cardiac safety of cetuximab and panitumumab, particularly as single agents, has not been investigated extensively. This trial was designed to specifically evaluate the cardiac safety of cetuximab and panitumumab as single therapy in Chinese chemotherapy-refractory metastatic colorectal cancer (mCRC) patients.
\end{abstract}

Patients and methods: Sixty-one patients received cetuximab at an initial dose of $400 \mathrm{mg} / \mathrm{m}^{2}$ intravenously over 120 minutes on day 1 (week 1), followed by a maintenance dose of $250 \mathrm{mg} / \mathrm{m}^{2}$ intravenously over 60 minutes on day 1 of each 7-day cycle. Forty-three patients received panitumumab at a dose of $6 \mathrm{mg} / \mathrm{kg}$ intravenously every 14 days. Routine laboratory tests and electrocardiogram (ECG) were performed at baseline, during therapy and after the treatment (4th and 10th months). The incidence of elevation of troponin I ultra (TNI Ultra), abnormal ECGs, cardiac events and noncardiac adverse events (AEs) were recorded and analyzed.

Results: The incidence of elevation of TNI Ultra between the two groups had no significance $(p=0.681)$, and TNI Ultra+ was observed more frequently in patients with metastases to more than three organs and they received fourth or above lines of chemotherapy. The most frequent abnormal ECG manifestations were nonspecific ST changes and QTc prolongation in the two groups. At 10 months after treatment, most of the abnormal ECG manifestations were reversed. The most common cardiac AEs of cetuximab and panitumumab included palpitations, dyspnea, chest pain and arrhythmias requiring treatment. Most of the events were mild and transient. The incidence of cardiac AEs had no significant difference between the two groups. Rash was still the most common noncardiac $\mathrm{AE}$ in both groups.

Conclusion: Cetuximab and panitumumab showed favorable cardiac safety as single agents for Chinese chemotherapy-refractory mCRC patients. But monitoring for cardiac AEs is still necessary throughout the entire treatment process.

Keywords: cetuximab, panitumumab, targeted therapy, colorectal cancer, toxicity, cardiac safety

\section{Introduction}

Cardiotoxicity is a common side effect of many agents for cancer therapy and presents a serious threat to the safety of patients. ${ }^{1}$ With the extensive clinical application of molecular targeting drugs, such as monoclonal antibodies (Mabs) and tyrosine kinase inhibitors, the cardiotoxicity issue has aroused more and more attention. ${ }^{2,3}$ Trastuzumab, a human epidermal growth factor receptor 2 inhibitor, has been demonstrated to have potential cardiotoxicity including decreased left ventricular ejection fraction. ${ }^{4}$ Moreover, QTc prolongation was reported to be associated with many tyrosine kinase inhibitors including lapatinib, imatinib, nilotinib, dasatinib, sunitinib and sorafenib. ${ }^{5,6}$ Drug-associated cardiotoxicity often emerges after heavy dose, which becomes the leading cause of 
discontinuity and withdrawal of the therapy. ${ }^{7}$ For many other targeted agents, the risk of cardiotoxicity remains uncertain.

Colorectal cancer (CRC) is the fourth leading cause of cancer death in the world. ${ }^{8} \mathrm{CRC}$ is frequently associated with abnormal overexpression of the epidermal growth factor receptor(EGFR). ${ }^{9,10}$ Anti-EGFR Mabs are usually used alone or in combination with cytotoxic agents as second- or third-line regimens. They are also used as monotherapy after chemotherapy failure.

Currently, cetuximab and panitumumab have been approved for the treatment of metastatic CRC (mCRC) patients with wild-type KRAS. ${ }^{11,12}$ The evidence of cardiotoxicity of cetuximab and panitumumab, particularly as a single agent after heavy chemotherapy, is still insufficient. This study was designed to specifically evaluate the cardiac safety of cetuximab and panitumumab as salvage monotherapy in Chinese chemotherapy-refractory mCRC patients.

\section{Patients and methods}

\section{Trial design}

This study was a Phase II trial conducted in Sun Yat-sen University Cancer Center (Guangzhou, China) that was designed to evaluate the cardiac safety of cetuximab and panitumumab as salvage monotherapy in Chinese chemotherapyrefractory $\mathrm{mCRC}$ patients. The protocol and all modifications were approved by the Institutional Review Board and the Ethics Committee of our center. The study strictly followed the Declaration of Helsinki and the Good Practice Guidelines. Written informed consent was obtained from every participant. The authenticity of this article has been validated by uploading the key raw data onto the Research Data Deposit public platform (www.researchdata.org.cn), with the approval RDD number as RDDA2017000430.

\section{Eligibility criteria}

The main eligibility criteria of inclusion were: age $\geq 18$ years; histologically or cytologically confirmed metastatic adenocarcinoma of the colon or rectum with wild-type RAS; measurable or non-measurable lesion according to Response Evaluation Criteria In Solid Tumors (version 1.1); ${ }^{13}$ an Eastern Cooperative Oncology Group performance status of $\leq 2$; a life expectancy of more than 3 months; disease progression (clinical or radiologic) or intolerance to irinotecan-based and oxaliplatin-based therapy; and having had previously received a thymidylate synthase inhibitor (including fluorouracil, capecitabine, raltitrexed) for CRC.

The main exclusion criteria were: previous anti-EGFR therapy in 3 months before enrollment; allergy to cetuximab or panitumumab; taking antitumor therapy within 30 days of enrollment; central nervous system metastases; clinically significant cardiovascular disease in 1 year before enrollment; active uncontrolled infection; other malignancies in the past 5 years with the exception of adequately treated carcinoma in situ of the cervix and squamous or basal cell carcinoma of the skin; inadequate hematologic function (absolute neutrophil count $<1.5 \times 10^{9} / \mathrm{L}$, platelet count $<75 \times 10^{9} / \mathrm{L}$, or hemoglobin $<80 \mathrm{~g} / \mathrm{L}$ ), inadequate renal function (creatinine $>1.5 \times$ upper limit of normal [ULN]), and inadequate hepatic function (total bilirubin $>1.5 \times \mathrm{ULN}$, or aspartate aminotransferase or alanine aminotransferase $>3 \times \mathrm{ULN}$ $[>5 \times$ ULN if the patient had liver metastases]).

\section{Intervention}

Sixty-one patients received cetuximab at an initial dose of $400 \mathrm{mg} / \mathrm{m}^{2}$ intravenously over 120 minutes on day 1 (week 1 ), followed by a maintenance dose of $250 \mathrm{mg} / \mathrm{m}^{2}$ intravenously over 60 minutes on day 1 of each 7-day cycle. Forty-three patients received panitumumab at a dose of $6 \mathrm{mg} / \mathrm{kg}$ intravenously every 14 days. The administration of recommended premedication (dexamethasone $10 \mathrm{mg}$ and cimetidine $200 \mathrm{mg}$ ) was required before every cycle. The maintenance of cetuximab or panitumumab was continued until disease progression, unacceptable toxicity, planned surgery, serious protocol violation or patient withdrawal.

\section{Assessments}

All patients were subjected to 15-lead electrocardiogram (ECG) in the baseline period ( $\leq 7$ days before enrollment) by a digital GE-MAC5500 machine, and it was repeated on the day before every cycle. ECG was also repeated at 4 and 10 months after initial therapy.

Blood samples for routine laboratory tests and ultrasensitive troponin I (TNI Ultra) were collected $1 \mathrm{~h}$ prior to cetuximab or panitumumab administration and $1 \mathrm{~h}$ after the drug infusion. The concentration of TNI Ultra was determined by a fluorometric enzyme immunoassay analyzer (Stratus CS; Dade Behring, Miami, FL, USA). The cutoff value of TNI Ultra was $0.78 \mathrm{ng} / \mathrm{mL}$. The elevation of TNI Ultra (TNI Ultra+) was defined as any value exceeding this value.

Cardiac adverse events (AEs; including nonspecific ST changes, sinus tachycardia, sinus bradycardia, negative T waves, poor R-wave progression, left ventricular hypertrophy, QTc prolongation, atrial premature beats and ventricular premature contraction) and noncardiac AEs (including allergic reaction, rash, onychia lateralis, diarrhea, nausea/vomiting, chest pain and fever) were recorded according to the National Cancer Institute's Common Terminology Criteria for Adverse Events (NCI-CTCAE) version 4.02.

Assessments of the efficacy of cetuximab or panitumumab were beyond the scope of this study. Disease 
response evaluated by radiologic approaches (computed tomography, magnetic resonance imaging or positron emission tomography-computed tomography) was documented at each evaluation visit (every 6-8 weeks), only for the purpose of confirming eligibility for continuing therapy.

\section{Statistical analyses}

The primary endpoint of this study was to determine and compare the cardiac AEs of cetuximab and panitumumab in the study population.

The secondary endpoint was to evaluate the noncardiac AEs of cetuximab and panitumumab.

All statistical tests were two-sided, and significance was assumed at $p<0.05$. Results are presented as mean $\pm \mathrm{SD}$, unless otherwise specified. The independent $t$-test was used for normally distributed continuous variables, and either the $\chi^{2}$ or the Fisher's exact test was used for categorical variables. Data were recorded and analyzed with SPSS (version 23) and GraphPad Prism (version 6.0).

\section{Results}

Between January 1, 2012, and December 31, 2015, 104 patients were enrolled in this study (Figure 1). Sixty-one patients received cetuximab and 43 received panitumumab. Baseline demographic and clinical characteristics are summarized in Table 1. In the cetuximab group, the patients received a median of 9 (range 4-28) cycles of therapy. In the panitumumab group, the patients received a median of 10 (range 3-32) cycles of therapy. The median follow-up duration was 14 months (range 10-17 months).

\section{TNI Ultra}

In the baseline screen, all patients had normal TNI Ultra level. TNI Ultra+ was observed in 21 patients from cetuximab group (34.4\%, mean value: $0.97 \pm 0.38 \mathrm{ng} / \mathrm{mL}$ ) and 17 patients from panitumumab group $(39.5 \%$, mean value: $0.85 \pm 0.27 \mathrm{ng} / \mathrm{mL})$. The incidence of TNI Ultra+ between the two groups had no significance $(p=0.681)$. The clinical characteristics of patients with TNI Ultra+ or TNI Ultra- are summarized in Table 1. In both groups, TNI Ultra+ was observed more frequently in patients with metastases to more than three organs and they received fourth or above lines of chemotherapy. Also, all the elevated TNI Ultra levels recovered at 10 months after therapy.

\section{Electrocardiogram}

In the baseline screen, there were two patients with nonspecific ST changes and two patients with QTc prolongation in cetuximab group, while there were three patients with QTc prolongation in panitumumab group. At 4 months after treatment, the most frequent abnormal ECG manifestations were nonspecific ST changes (20 cases, 32.7\%) and QTc prolongation (22 cases, 36.1\%) in cetuximab group. Similarly, the most frequent abnormal ECG manifestations were nonspecific ST changes (11 cases, 25.6\%) and QTc prolongation ( 7 cases, $16.3 \%$ ) in panitumumab group. There was no significant difference in the incidence of nonspecific ST changes ( $p=0.516$ ) between the two groups. But the incidence of QTc prolongation was higher in cetuximab group than in panitumumab group ( $p=0.029$ ).

At 10 months after treatment, most of the abnormal ECG manifestations reversed. There were still three patients with

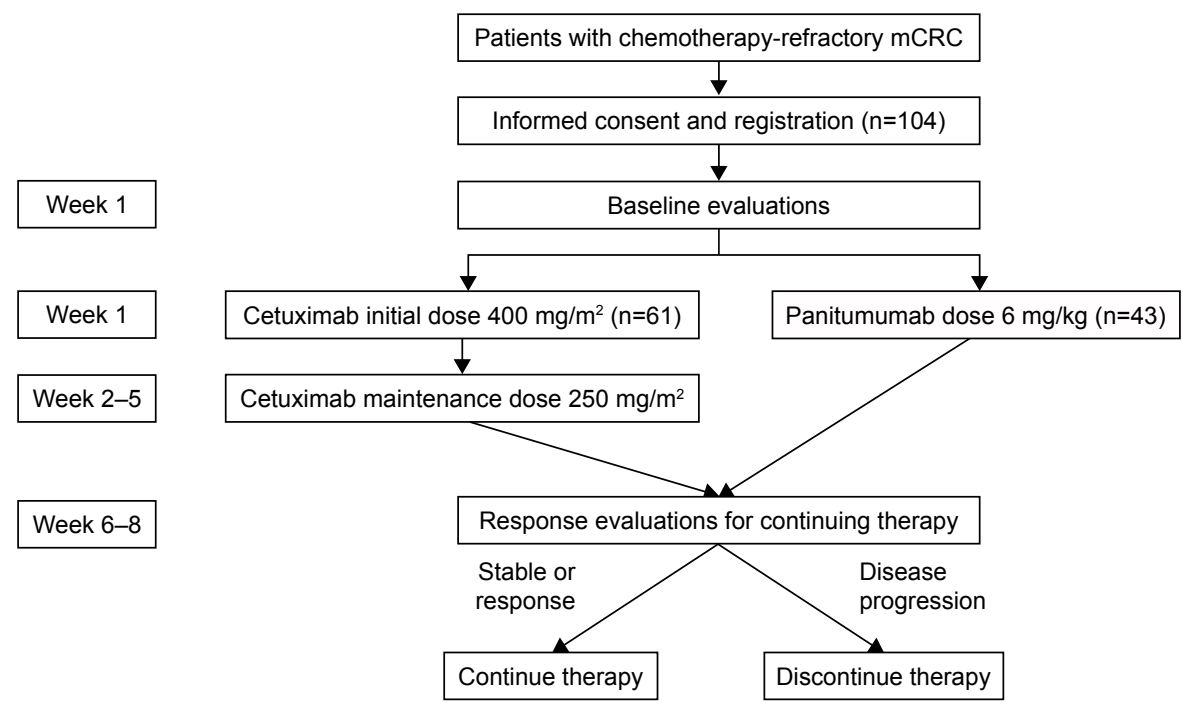

Figure I Study flowchart

Abbreviation: $\mathrm{mCRC}$, metastatic colorectal cancer. 
Table I Clinical characteristics of patients in cetuximab and panitumumab groups (analyzed with TNI Ultra level)

\begin{tabular}{|c|c|c|c|c|c|c|}
\hline \multirow[t]{3}{*}{ Characteristic } & \multicolumn{3}{|c|}{ Cetuximab $(n=6 I)$} & \multicolumn{3}{|c|}{ Panitumumab $(n=43)$} \\
\hline & \multirow{2}{*}{$\begin{array}{l}\text { TNI Ultra }+ \\
\mathrm{n}\end{array}$} & \multirow{2}{*}{$\frac{\text { TNI Ultra- }}{n}$} & \multirow[t]{2}{*}{$p$-value } & \multirow{2}{*}{$\frac{\text { TNI Ultra }+}{\mathbf{n}}$} & \multirow{2}{*}{$\frac{\text { TNI Ultra- }}{\mathrm{n}}$} & \multirow[t]{2}{*}{$p$-value } \\
\hline & & & & & & \\
\hline Age, years & $56.7 \pm 1.9$ & $53.5 \pm 2.3$ & 0.168 & $53.1 \pm 4.2$ & $51.9 \pm 2.8$ & 0.325 \\
\hline \multicolumn{7}{|l|}{ Gender } \\
\hline Male & 13 & 28 & 0.574 & 12 & 18 & 1.000 \\
\hline Female & 8 & 12 & & 5 & 8 & \\
\hline \multicolumn{7}{|l|}{ ECOG performance status } \\
\hline 0 & 3 & 15 & 0.105 & 5 & 9 & 0.847 \\
\hline 1 & 13 & 21 & & 10 & 13 & \\
\hline 2 & 5 & 4 & & 2 & 4 & \\
\hline \multicolumn{7}{|l|}{ Primary tumor location } \\
\hline Colon & 12 & 19 & 0.592 & 7 & 13 & 0.756 \\
\hline Rectum & 9 & 21 & & 10 & 13 & \\
\hline \multicolumn{7}{|l|}{ Histology (adenocarcinoma) } \\
\hline Well differentiated & 0 & 3 & 0.677 & 0 & 2 & 0.707 \\
\hline Moderately differentiated & 17 & 31 & & 14 & 20 & \\
\hline Poorly differentiated & 4 & 6 & & 3 & 4 & \\
\hline \multicolumn{7}{|l|}{ Metastatic site } \\
\hline Liver & 15 & 19 & 0.847 & 9 & 13 & 0.947 \\
\hline Lung & 13 & 14 & & 6 & 10 & \\
\hline Lymph nodes & 6 & 5 & & 3 & 5 & \\
\hline Bones & 2 & 4 & & I & 3 & \\
\hline \multicolumn{7}{|l|}{ Organs affected } \\
\hline 2 & 5 & 22 & 0.029 & 3 & 15 & 0.026 \\
\hline$\geq 3$ & 16 & 18 & & 14 & 12 & \\
\hline \multicolumn{7}{|l|}{ Lines of chemotherapy } \\
\hline Third line & 5 & 23 & 0.016 & 3 & 14 & 0.018 \\
\hline Fourth and above lines & 16 & 17 & & 14 & 12 & \\
\hline
\end{tabular}

Notes: Percentages do not always add up to $100 \%$ because of rounding errors and overlapping data. $p$-values $<0.05$ are shown in bold.

Abbreviations: ECOG, Eastern Cooperative Oncology Group; TNI, troponin I.

nonspecific ST changes and three patients with QTc prolongation in cetuximab group, while there were two patients with nonspecific ST changes and four patients with QTc prolongation in panitumumab group. There was no significant difference in the incidence of nonspecific ST changes ( $p=1.000)$ and QTc prolongation $(p=0.444)$ between the two groups (Table 2; Figure 2).

\section{Cardiac AEs}

Overall, 32 cardiac AEs cetuximab group and 18 cardiac AEs in panitumumab group were observed (Table 3 ). There were no severe events such as acute coronary syndrome, heart failure and cardiac death in either group. Except for one case of grade 3 chest pain which needed intervention (oxygen inhalation, ECG monitoring and administration of nitroglycerin),

Table 2 Summary of ECG abnormalities in cetuximab and panitumumab groups

\begin{tabular}{|c|c|c|c|c|c|c|}
\hline \multirow[t]{3}{*}{ ECG manifestation } & \multicolumn{3}{|c|}{ Cetuximab $(n=6 I)$} & \multicolumn{3}{|c|}{ Panitumumab $(n=43)$} \\
\hline & \multirow{2}{*}{ Baseline } & \multirow{2}{*}{$\begin{array}{l}4 \text { months after } \\
\text { treatment } \\
n\end{array}$} & \multirow{2}{*}{$\begin{array}{l}10 \text { months after } \\
\text { treatment } \\
\mathrm{n}\end{array}$} & \multirow{2}{*}{$\begin{array}{l}\text { Baseline } \\
\mathbf{n}\end{array}$} & \multirow{2}{*}{$\begin{array}{l}4 \text { months after } \\
\text { treatment } \\
\mathrm{n}\end{array}$} & \multirow{2}{*}{$\begin{array}{l}10 \text { months } \\
\text { after treatment } \\
n\end{array}$} \\
\hline & & & & & & \\
\hline Nonspecific ST changes & 2 & 20 & 3 & 0 & 11 & 2 \\
\hline Sinus tachycardia & 0 & 6 & 0 & 0 & 2 & 0 \\
\hline Sinus bradycardia & 0 & I & 0 & 0 & 3 & 0 \\
\hline Negative T waves & 0 & 6 & 0 & 0 & 4 & 0 \\
\hline Poor R-wave progression & 0 & 3 & 0 & 0 & 2 & 0 \\
\hline LV hypertrophy & 0 & 4 & 2 & 0 & 3 & 1 \\
\hline QTc prolongation & 2 & 22 & 3 & 3 & 7 & 4 \\
\hline Atrial premature beats & 0 & 2 & 0 & 0 & 1 & 0 \\
\hline Ventricular premature contraction & 0 & 3 & 0 & 0 & 2 & 0 \\
\hline
\end{tabular}

Abbreviations: ECG, electrocardiography; LV, left ventricular. 



Figure 2 The incidence of abnormal ECG in the two groups.

Abbreviations: ECG, electrocardiogram; LV, left ventricular.

all events were mild and could recover without causing severe damage. The incidence of cardiac AEs had no significant difference between the two groups, which shows that cetuximab and panitumumab have similar cardiac safety profiles.

\section{Noncardiac AEs}

Rash was the most frequent noncardiac $\mathrm{AE}$ in both groups during the therapy. There were 36 cases $(59.1 \%)$ of rash in

Table 3 Common cardiac AEs in cetuximab and panitumumab groups

\begin{tabular}{|c|c|c|c|c|}
\hline \multirow[t]{3}{*}{ Cardiac AEs } & \multicolumn{2}{|c|}{ Cetuximab } & \multicolumn{2}{|c|}{ Panitumumab } \\
\hline & $\begin{array}{l}\text { Any } \\
\text { grade }\end{array}$ & $\begin{array}{l}\text { Grade } \\
3 / 4\end{array}$ & $\begin{array}{l}\text { Any } \\
\text { grade }\end{array}$ & $\begin{array}{l}\text { Grade } \\
3 / 4\end{array}$ \\
\hline & $n$ & $n$ & $n$ & $n$ \\
\hline Chest pain & 5 & 1 & 3 & 0 \\
\hline Palpitations & 16 & 0 & 8 & 0 \\
\hline Dyspnea & 8 & 0 & 5 & 0 \\
\hline $\begin{array}{l}\text { Arrhythmias requiring } \\
\text { treatment }\end{array}$ & 3 & 0 & 2 & 0 \\
\hline
\end{tabular}

Abbreviation: AEs, adverse events. cetuximab group; 11 of them were grade 3/4. Also, there were 30 cases $(69.8 \%)$ of rash in panitumumab group and 9 of them were grade $3 / 4$. Other frequent events included fever, fatigue and nausea/vomiting. Most of the AEs could recover after symptomatic treatment. The hematologic toxicities were rare and mild (Table 4).

Table 4 Common noncardiac AEs in cetuximab and panitumumab groups

\begin{tabular}{|c|c|c|c|c|}
\hline \multirow[t]{3}{*}{ Noncardiac AEs } & \multicolumn{2}{|c|}{ Cetuximab } & \multicolumn{2}{|c|}{ Panitumumab } \\
\hline & $\begin{array}{l}\text { Any } \\
\text { grade }\end{array}$ & $\begin{array}{l}\text { Grade } \\
3 / 4\end{array}$ & $\begin{array}{l}\text { Any } \\
\text { grade }\end{array}$ & $\begin{array}{l}\text { Grade } \\
3 / 4\end{array}$ \\
\hline & $\mathbf{n}$ & $\mathbf{n}$ & $\mathbf{n}$ & $\mathbf{n}$ \\
\hline Rash & 36 & II & 30 & 9 \\
\hline Allergic reaction & 2 & 0 & 0 & 0 \\
\hline Onychia lateralis & 6 & 3 & 2 & 0 \\
\hline Diarrhea & 5 & 0 & 8 & 0 \\
\hline Nausea/vomiting & 9 & 0 & 5 & 0 \\
\hline Fever & 15 & 0 & 5 & 0 \\
\hline Fatigue & II & 0 & 7 & 0 \\
\hline
\end{tabular}

Abbreviation: AEs, adverse events. 


\section{Discussion}

Targeted therapies for cancer have developed rapidly over the last two decades. Anti-EGFR antibodies were considered as appropriate options for $\mathrm{mCRC}$ patients who showed failure of response to chemotherapy. ${ }^{13,14}$ The use of anti-EGFR Mabs in mCRC patients with wild-type RAS gene has significantly improved the prognosis, even as a single agent in chemotherapy-refractory patients. ${ }^{15,16}$ Currently, cetuximab and panitumumab are the two most common anti-EGFR Mabs for the treatment of mCRC.

Cetuximab is a chimeric Mab that can bind to the extracellular domain of EGFR in its inactive state; it competes for receptor binding by occluding the ligand-binding region, thereby blocking the ligand-induced EGFR tyrosine kinase activation and downstream signal pathway. Panitumumab is a recombinant human $\mathrm{IgG} 2 \mathrm{Mab}$ that binds specifically to EGFR. The ASPECCT trial showed that cetuximab and panitumumab were similar in activity. ${ }^{17}$

Although cardiac AEs are not the main side effects of EGFR Mabs, ${ }^{18}$ Boku et al reported the incidence rate of cardiac disorders as $0.2 \%$ in 3,085 Japanese mCRC patients treated with panitumumab as monotherapy (40.7\%) or combination therapy $(59.4 \%) .{ }^{19}$ Notably, monotherapy of EGFR Mabs often followed previous intensive chemotherapy. Although cardiac toxicities are not common with the main cytotoxic agents used for mCRC, such as oxaliplatin, irinotecan and capecitabine, it is uncertain if the accumulative nonspecific toxicities of previous chemotherapy will increase the risk of cardiac AEs of EGFR Mabs.

In this study, we employed active surveillance of TNI Ultra and ECG for the evaluation of potential cardiac damages in Chinese chemotherapy-refractory $\mathrm{mCRC}$ patients treated with cetuximab and panitumumab as single therapy. In the study population, all patients had normal TNI Ultra level before cetuximab and panitumumab treatment. TNI Ultra+ was observed in 21 patients from cetuximab group (34.4\%, mean value: $0.97 \pm 0.38 \mathrm{ng} / \mathrm{mL}$ ) and 17 patients from panitumumab group (39.5\%, mean value: $0.85 \pm 0.27 \mathrm{ng} / \mathrm{mL})$. The analysis of association of TNI Ultra+ with clinicopathologic characteristics revealed that TNI Ultra+ was observed more frequently in patients with metastases to more than three organs and they received fourth or above lines of chemotherapy. It suggested that the higher incidence of TNI Ultra+ might partially be attributed to the relative advanced stage and excessive chemotherapy. Also, the increased TNI Ultra levels in all patients reverted to normal at 10 months after therapy, which indicated that the increase of TNI Ultra by cetuximab and panitumumab was transient and reversible.
As to the ECG evaluation during cetuximab and panitumumab therapy, the most frequent abnormal ECG manifestations were nonspecific ST changes (20 cases, 32.7\%) and QTc prolongation (22 cases, 36.1\%) in cetuximab group and nonspecific ST changes (11 cases, 25.6\%) and QTc prolongation ( 7 cases, $16.3 \%$ ) in panitumumab group. There was no significant difference in the incidence of nonspecific ST changes ( $p=0.516$ ) between the two groups. But the incidence of QTc prolongation was higher in cetuximab group than in panitumumab group ( $p=0.029)$. Nonspecific ST changes usually indicate the presence of myocardial ischemia or injury. ${ }^{20,21}$ QTc prolongation is one of the severe abnormalities of ECG which may lead to malignant ventricular tachyarrhythmias such as torsades de pointes, which can quickly progress to ventricular fibrillation and sudden death. ${ }^{22,23}$ At 10 months after treatment, there were still three patients with nonspecific ST changes and three patients with QTc prolongation in cetuximab group, while there were two patients with nonspecific ST changes and four patients with QTc prolongation in panitumumab group. This result might suggest that most of the nonspecific ST changes and QTc prolongation caused by cetuximab and panitumumab therapy were reversible; the persistence of such abnormalities needs further follow-up and attention.

The most common cardiac AEs of cetuximab and panitumumab in our study included palpitations, dyspnea, chest pain and arrhythmias requiring treatment. Most of the events were mild and transient. Palpitations and dyspnea usually occurred with rapid infusion of drugs and could be relieved with adjustment of infusion speed. The most common noncardiac AEs in our study were skin rashes; some of them were grade 3/4 and needed antianaphylaxis agents and/or corticosteroid ointment. Other noncardiac AEs included diarrhea, fever and nausea/vomiting, which were consistent with the known side effects of anti-EGFR Mabs.

\section{Conclusion}

Together, cetuximab and panitumumab showed favorable cardiac safety as a single agent for Chinese chemotherapyrefractory mCRC patients in our study. But enough alert and regular monitoring for cardiac AEs are still necessary for the benefit of patients, especially in patients with multiple organ metastases and heavy previous chemotherapy. The surveillance of TNI Ultra and ECG might be an appropriate approach for the timely detection and intervention of such AEs.

\section{Acknowledgment}

This work was supported by the Guangdong Medical Research Foundation (A2016156). 


\section{Disclosure}

The authors report no conflicts of interest in this work.

\section{References}

1. Yeh ET. Cardiotoxicity induced by chemotherapy and antibody therapy. Annu Rev Med. 2006;57:485-498.

2. Albini A, Pennesi G, Donatelli F, Cammarota R, De Flora S, Noonan DM. Cardiotoxicity of anticancer drugs: the need for cardio-oncology and cardio-oncological prevention. J Natl Cancer Inst. 2010;102(1): $14-25$.

3. Strevel EL, Ing DJ, Siu LL. Molecularly targeted oncology therapeutics and prolongation of the QT interval. J Clin Oncol. 2007;25(22): 3362-3371.

4. Yeh ET, Bickford CL. Cardiovascular complications of cancer therapy: incidence, pathogenesis, diagnosis, and management. J Am Coll Cardiol. 2009;53(24):2231-2247.

5. Raschi E, De Ponti F. Cardiovascular toxicity of anticancer-targeted therapy: emerging issues in the era of cardio-oncology. Intern Emerg Med. 2012;7(2):113-131.

6. Raschi E, Vasina V, Ursino MG, Boriani G, Martoni A, De Ponti F. Anticancer drugs and cardiotoxicity: Insights and perspectives in the era of targeted therapy. Pharmacol Ther. 2010;125(2):196-218.

7. Deeken JF, Shimkus B, Liem A, et al. Evaluation of the relationship between cetuximab therapy and corrected QT interval changes in patients with advanced malignancies from solid tumors. Cancer Chemother Pharmacol. 2013;71(6):1473-1483.

8. Frestad D, Perner A, Pedersen UG. Acute onset and rapid progression of multiple organ failure in a young adult with undiagnosed disseminated colonic adenocarcinoma. BMJ Case Rep. 2014;2014:bcr2014205002.

9. Gullick WJ. Prevalence of aberrant expression of the epidermal growth factor receptor in human cancers. Br Med Bull. 1991;47(1):87-98.

10. Herbst RS, Shin DM. Monoclonal antibodies to target epidermal growth factor receptor-positive tumors: a new paradigm for cancer therapy. Cancer. 2002;94(5):1593-1611.

11. Karapetis CS, Khambata-Ford S, Jonker DJ, et al. K-ras mutations and benefit from cetuximab in advanced colorectal cancer. $N$ Engl $J$ Med. 2008;359(17):1757-1765.

12. Amado RG, Wolf M, Peeters M, et al. Wild-type KRAS is required for panitumumab efficacy in patients with metastatic colorectal cancer. J Clin Oncol. 2008;26(10):1626-1634.
13. Gill S, Dowden S, Colwell B, Collins LL, Berry S. Navigating later lines of treatment for advanced colorectal cancer - optimizing targeted biological therapies to improve outcomes. Cancer Treat Rev. 2014; 40(10):1171-1181.

14. Chibaudel B, Bonnetain F, Tournigand C, et al. STRATEGIC-1: A multiple-lines, randomized, open-label GERCOR phase III study in patients with unresectable wild-type RAS metastatic colorectal cancer. BMC Cancer. 2015;15:496.

15. Cunningham D, Humblet $Y$, Siena S, et al. Cetuximab monotherapy and cetuximab plus irinotecan in irinotecan-refractory metastatic colorectal cancer. N Engl J Med. 2004;351(4):337-345.

16. De Roock W, Claes B, Bernasconi D, et al. Effects of KRAS, BRAF, NRAS, and PIK3CA mutations on the efficacy of cetuximab plus chemotherapy in chemotherapy-refractory metastatic colorectal cancer: a retrospective consortium analysis. Lancet Oncol. 2010;11(8): 753-762.

17. Price TJ, Peeters M, Kim TW, et al. Panitumumab versus cetuximab in patients with chemotherapy-refractory wild-type KRAS exon 2 metastatic colorectal cancer (ASPECCT): a randomised, multicentre, open-label, non-inferiority phase 3 study. Lancet Oncol. 2014;15(6): 569-579.

18. Martinelli E, De Palma R, Orditura M, De Vita F, Ciardiello F. Antiepidermal growth factor receptor monoclonal antibodies in cancer therapy. Clin Exp Immunol. 2009;158(1):1-9.

19. Boku N, Sugihara K, Kitagawa Y, et al. Panitumumab in Japanese patients with unresectable colorectal cancer: a post-marketing surveillance study of 3085 patients. Jpn J Clin Oncol. 2014;44(3): 214-223.

20. Rasouli ML, Ellestad MH. Usefulness of ST depression in ventricular premature complexes to predict myocardial ischemia. Am J Cardiol. 2001;87(7):891-894.

21. Hopenfeld B, Stinstra JG, Macleod RS. Mechanism for ST depression associated with contiguous subendocardial ischemia. J Cardiovasc Electrophysiol. 2004;15(10):1200-1206.

22. Belardinelli L, Antzelevitch C, Vos MA. Assessing predictors of drug-induced torsade de pointes. Trends Pharmacol Sci. 2003;24(12): 619-625.

23. Belardinelli L, Shryock JC, Wu L, Song Y. Use of preclinical assays to predict risk of drug-induced torsades de pointes. Heart Rhythm. 2005;2(2 Suppl):S16-S22.
OncoTargets and Therapy

\section{Publish your work in this journal}

OncoTargets and Therapy is an international, peer-reviewed, open access journal focusing on the pathological basis of all cancers, potential targets for therapy and treatment protocols employed to improve the management of cancer patients. The journal also focuses on the impact of management programs and new therapeutic agents and protocols on

\section{Dovepress}

patient perspectives such as quality of life, adherence and satisfaction. The manuscript management system is completely online and includes a very quick and fair peer-review system, which is all easy to use. Visit http://www.dovepress.com/testimonials.php to read real quotes from published authors. 